Research Paper

\title{
Subcutaneous Radiographic Measurement: A Marker to Evaluate Surgical Site Infection Risk in Elderly Hip Fracture Patients
}

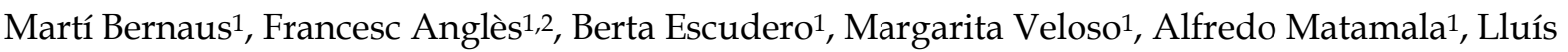 \\ Font-Vizcarra $^{1}$ 迥 \\ 1. Department of Traumatology and Orthopedic Surgery, Hospital Universitari Mútua Terrassa, Barcelona, Spain \\ 2. Department of Surgery, Universitat de Barcelona \\ $\triangle$ Corresponding author: Lluís Font-Vizcarra, Email: 1lfont@mutuaterrassa.cat; Telephone: +34 9373650 50; Address: Plaça Dr. Robert n5, 08221, Terrassa, \\ Barcelona, Spain. \\ (C) Ivyspring International Publisher. This is an open access article distributed under the terms of the Creative Commons Attribution (CC BY-NC) license \\ (https://creativecommons.org/licenses/by-nc/4.0/). See http://ivyspring.com/terms for full terms and conditions.
}

Received: 2018.09.24; Accepted: 2018.12.12; Published: 2019.01.24

\begin{abstract}
Objectives: Obesity is a documented comorbidity that is prevalent in the elderly population and a known predictor for surgical site infection (SSI). Body mass index is a convenient method to classify obesity, but it fails to account for fat distribution. The objective of our study was to evaluate the association between surgical site infection and a subcutaneous radiographic measurement (SRM) in elderly hip fracture patients.

Materials and Methods: A retrospective case-control study was conducted to compare SRMs at the hip in patients diagnosed with surgical site infection after hip fracture surgery with patients that were not diagnosed with surgical site infection. Each case was matched to two controls. An SRM was defined as the distance from the tip of the greater trochanter to the skin following a perpendicular line to the femoral diaphysis in anteroposterior hip radiographs. Clinical diagnosis of acute surgical site infection was based on Tsukayama criteria.

Results: Patients with an SRM greater than $6.27 \mathrm{~cm}$ had a 7 -fold increase in the odds of surgical site infection $(\mathrm{OR}=7.42$, 95\% Confidence Interval $(\mathrm{Cl})=3.01-18.28, \mathrm{p}<0.001)$ compared to those with smaller measurements. The odds ratio (OR) for infection of patients with an ASA score of 3 was $15.82(95 \%$ $\mathrm{Cl}=5.11$-48.9, $\mathrm{P}$-value<0.001)

A statistically significant difference between cases and controls was also found when SRM at the hip was analyzed as a continuous variable. Patients with an infection had a $2.24 \mathrm{~cm}(95 \% \mathrm{Cl}=1.59-2.90 ; \mathrm{p}<0.001)$ greater mean SRM.

Conclusion: Results of our study suggest an association between the SRM at the hip and the risk of SSI in elderly patients with surgically treated hip fractures. SRM may be a helpful tool for evaluating the risk of SSI in elderly hip fracture patients.
\end{abstract}

Key words: hip fracture, elderly, surgical site infection, radiographic measurement

\section{Introduction}

Surgical site infections (SSIs) in orthopedic trauma account for $11 \%$ of all nosocomial infections in elderly patients [1]. Diagnosis and treatment of SSIs have an important economic impact on healthcare systems and are known to have adverse effects on outcomes for patients. Prevalence estimates have shown an increase in hip fractures in future years and it is fitting to assume an increase in SSIs for patients with hip fractures, as SSIs after hip fractures occur in $5-10 \%$ of patients [2]. Documented co-morbidities that are prevalent in the elderly population and are known predictors of surgical site infections include diabetes, 
immunosuppression, renal or hepatic diseases, age, alcohol or tobacco use, vascular insufficiency, and obesity [2-7].

Although exact physiopathology of obesity as a risk factor for surgical site infection has not been fully described, some recent studies involving total hip replacement patients have addressed this issue [8-10]. Possible explanations associating obesity and infection suggest that local and systemic deposition of antibiotic prophylaxis may be altered in obese patients. Markedly reduced tissue penetration of antibiotics in obese patients could jeopardize optimal minimal inhibitory concentration during surgery, potentially increasing the risk of surgical site infection [11,12]. Other explanations include technical difficulties for surgeons, decreased blood flow and oxygen tension, increased operating times and comorbidities related to obesity $[13,14]$.

Obesity has been classically quantified and classified by means of Body Mass Index (BMI). There are many confounders that can potentially affect BMI and outcomes [15]. BMI does not account for fat distribution and is not capable of distinguishing muscle tissue from adipose tissue. There is a growing interest in fat distribution, rather than BMI, as an important factor for evaluating the risk of surgical infection. Some studies in general surgery and lumbar spine surgery have postulated that local fat thickness at the incision site may increase the risk of surgical site infection [16]. However, the exact measurement of local soft tissue thickness can only be assessed intraoperatively. For this reason, preoperative detection of patients at a higher risk of surgical site infection is challenging and preventive strategies are limited. The aim of our study was to evaluate the possible association between subcutaneous radiographic measurement (SRM) and the risk of SSI. To the best of our knowledge, there are no similar studies in hip fracture patients. This study hypothesizes that greater measurements of local soft tissue at the hip, measured with radiographs, are associated with an increased risk of surgical site infection in elderly patients (age>65) with a hip fracture.

\section{Methods}

A retrospective case-control study was conducted to compare SRMs at the hip in patients diagnosed with surgical site infection after hip fracture surgery with patients that were not diagnosed with surgical site infection. For this study we included all patients diagnosed with an acute SSI admitted to our hospital for hip fracture surgery from 2012 to 2016.

Included patients were diagnosed with intra or extracapsular fractures and underwent either internal fixation or hip replacement procedures according to a Geriatric Trauma Unit's indication. All patients were operated under the supervision of an associate surgeon. Antibiotic prophylaxis was performed with a second-generation cephalosporin for non-allergic patients, clindamycin for allergic patients and teicoplanin for patients with colonization with Methicillin-Resistant Staphylococcus aureus. Posterolateral approaches were performed in arthroplasty patients and cemented stems with gentamycin were used in all cases. No drains were used.

The study was approved by the ethics committee at our institution and informed consent was obtained for all participants.

\section{Patients and variables}

Cases were defined as patients diagnosed with an acute SSI after surgical treatment of a femoral fracture.

To minimize selection bias, each case was matched to two controls using the following methodology: patients of the same sex, the same fracture classification (intracapsular or extracapsular) and procedure (total hip arthroplasty (THA), hemiarthroplasty or internal fixation) with an age variation of + /- five years and nearest in surgery date to the case were selected. All participants had high-quality anteroposterior hip x-rays available and were followed for at least three months.

The principal variables collected were SRM and acute SSI. Secondary variables collected included age, gender, fracture classification (intracapsular or extracapsular), treatment (Intramedullary (IM) nail, dynamic hip screw (DHS) hemiarthroplasty, THA, American Society of Anesthesiologists (ASA) physical status classification and comorbidities such as rheumatoid arthritis, diabetes mellitus, chronic renal insufficiency, liver cirrhosis, current use of corticosteroids and dementia.

\section{Criteria for acute SSI Diagnosis}

The clinical diagnosis of acute SSI was based on Tsukayama criteria [17]: local inflammatory signs, purulent drainage through the wound, and elevated C-reactive protein during the first 4 weeks after the index surgery. A surgical debridement was performed in all patients with clinical diagnosis of acute SSI. During the surgery, several different samples were taken to obtain a microbiological diagnosis. In cases with arthroplasties, modular components were changed.

\section{Methodology for assessment of SRM}

Once cases and controls were identified and included, two different blinded orthopedic surgeons measured the distance from the tip of the greater 
trochanter to the skin following a perpendicular line to the femoral diaphysis in anteroposterior hip radiographs. (Fig 1) The final SRM was defined as the mean of each observer's evaluation. Standardized anteroposterior pelvis $\mathrm{x}$-rays in the supine position and $15-25^{\circ}$ of internal rotation of the hips were obtained for all patients.

No previously calibrated $\mathrm{x}$-rays were available. In order to obtain precise measurements, the first step required calibrating $\mathrm{x}$-rays. In patients with IM nails, the known diameter of the nail was introduced into the TraumaCad x-ray viewer program (BrainLab Ltd., Petach-Tikva, Israel) and set as a reference. Once this calibration process was performed, we proceeded to obtain the measurement from the tip of the greater trochanter to the skin as previously described. In patients with a hemiarthroplasty or total hip arthroplasty, the same procedure was followed setting the known diameter of the dome/cup component as a reference. In patients with a DHS, the known cephalic screw length was used as a reference. These measurements were taken in the first postoperative $x$-ray available.

\section{Statistical analysis}

Qualitative or ordinal variables were described by means of absolute frequencies or percentages while quantitative variables were described as mean or median values when necessary. Univariate analyses were performed using T-Student test or Chi-square Test for quantitative and dichotomous variables. SRM was analyzed as a continuous variable and as a dichotomous variable using the median of our measurements as a threshold. ASA was also dichotomized in two groups and we compared patients that were ASA 3 with those that were ASA 1 or 2. The Intraclass Correlation Coefficient (ICC) was calculated to evaluate the grade of correlation of SRMs between the two blinded observers.

Univariate and a multivariate logistic regression model were used to estimate the risk of presenting infection as odds ratio (OR) and 95\% confidence intervals (CI).

All analyses were performed using SPSS for MAC v22 (SPSS Inc., Chicago, IL, USA) Level of statistical significance was established at $\mathrm{p} \leq 0.05$ (two-tailed).

\section{Results}

A total of 120 participants (40 cases and 80 controls) were included in this analysis. There were 90(75\%) female patients and 30(25\%) male patients. The mean age of participants was 82 years. $78(65 \%)$ patients were diagnosed and treated for extracapsular fractures. $54(45 \%)$ patients were treated with a short IM nail, 15(12.5\%) with a long IM nail and 9(7.5\%) with a sliding hip screw. $42(35 \%)$ patients were diagnosed and treated for intracapsular fractures. $26(21.7 \%)$ patients were treated with a bipolar hemiarthroplasty, $12(10 \%)$ with a unipolar hemiarthroplasty and $4(3.3 \%)$ with a total hip replacement. Distribution of implants in the infection group was: 18 short nails (45\%), 5 long nails (12.5\%), 3 sliding hip screws $(7.5 \%), 9$ bipolar hemiarthroplasties (22.5\%), 4 monopolar hemiarthroplasties $(10 \%)$ and one total hip replacement $(2.5 \%)$. Pathogens isolated in cultures were Methicillin Sensitive S. Aureus (MSSA) (30\%), mixed flora $(33 \%)$, Gram- negative rods $(20 \%)$, coagulase-negative staphylococci $(8 \%)$, Cutibacterium acnes (5\%) and Methicillin-resistant S. Aureus (MRSA) (2\%). One patient had negative cultures.

In order to assess the reliability of measurements between the two observers, an additional analysis was performed using the Intraclass Correlation Coefficient (ICC) score. The overall agreement between observers was good (ICC $=0.822,95 \%$ CI: 0.754-0.873, p<0.001).

In the univariate analysis, distribution of previously studied risk factors between cases and controls did not show significant differences except for patients with an ASA score of 3 and subcutaneous radiographic measurement. (Table 1 ) The odds ratio (OR) for infection of
Figure 1. The images above show calibration procedures and subcutaneous radiographic measurement (SRM) assessment. Figure 1A shows measurement for hemiarthroplasty and figure 1B for an IM nail. First, a parallel lin to the femoral diaphysis was drawn. In figure 1A, a known measure was introduced as a reference for the dome component (in this case $46 \mathrm{~mm}$ ). In figure $1 \mathrm{~B}$, the nail diameter was used as a reference $(11 \mathrm{~mm})$. Following this calibration procedure, a perpendicular line to the femoral diaphysis was drawn from the tip of the greater trochanter and the distance to the skin from this point was measured. 
patients with an ASA score of 3 was 15.82 (95\% Confidence Interval $(\mathrm{CI})=5.11-48.9$, $\mathrm{p}$-value $<0.001)$.

The median for the SRM at the hip for all patients was $6.27 \mathrm{~cm}$. When participants were separated into two groups based on this threshold, a significant association between SSI and SRM was found for patients with greater measurements (Table 1). Patients with an SRM at the hip greater than $6.27 \mathrm{~cm}$ had a 7 -fold increase in the odds of surgical site infection $(\mathrm{OR}=7.42,95 \% \mathrm{CI}=3.01-18.28, \mathrm{p}<0.001)$ compared to those with smaller measurements. A statistically significant difference between cases and controls was also found when SRM was analyzed as a continuous variable. Patients with an infection had, on average, a 2.24cm (95\% CI: 1.59-2.90; $p<0.001$ ) greater mean SRM.

Finally, we performed a multivariate logistic regression model to investigate the association between surgical site infection and the variables that showed association in the univariate model (ASA score of 3 and SRM). This analysis showed an independent association between surgical site infection and ASA 3 score $(\mathrm{OR}=10.12,95 \% \mathrm{CI}$ 3.06-33.44, $\mathrm{p}<0.001)$ as well as an association between surgical site infection and SRM as a continuous variable $(\mathrm{OR}=1.92,95 \%$ CI 1.38-2.67, $\mathrm{p}>0.001)$.

Table 1. Univariate analysis results

\begin{tabular}{llll}
\hline & Infection & & P-value \\
\cline { 2 - 4 } & No $(\mathbf{n}=\mathbf{8 0})$ & Yes $(\mathbf{n}=\mathbf{4 0 )}$ & \\
\hline Mean Age, year (SD) & $82.66(6.70)$ & $80.93(8.74)$ & 0.26 \\
Female gender & $60(75 \%)$ & $30(75 \%)$ & 1.0 \\
Diabetes mellitus & $25(31.3 \%)$ & $13(32.5 \%)$ & 1.0 \\
ASA score 3 & $29(36.3 \%)$ & $36(90 \%)$ & $<0,001$ \\
Rheumatoid arthritis & $1(1.3 \%)$ & $0(0 \%)$ & 1 \\
Chronic renal Insufficiency & $17(21.3 \%)$ & $5(12.5 \%)$ & 0.32 \\
Liver cirrhosis & $5(6.3 \%)$ & $1(2.5 \%)$ & 0.662 \\
Use of corticoids & $9(11.3 \%)$ & $1(2.5 \%)$ & 0.162 \\
Dementia & $23(28.8 \%)$ & $8(20 \%)$ & 0.282 \\
Mean Subcutaneous Radiographic & $5.78(1.52)$ & $8.03(2.01)$ & $<0,001$ \\
Measurement cm(SD) & & & \\
Subcutaneous Radiographic & $28(35 \%)$ & $32(80 \%)$ & $<0.001$ \\
Measurement $>6.27$ cm & & & \\
\hline SD: Standard Deviation & & &
\end{tabular}

\section{Discussion}

Identifying patients who are at high risk for complications is a critical aspect of surgical decision making. In the subgroup of elderly patients, frailty and pre-existing comorbidities increase the risk of surgical complications [18]. For this reason, it is of utmost importance for surgeons and patients to preoperatively evaluate the potential risk of SSI.

The relationship between obesity, body mass index (BMI), local subcutaneous measurement and risk of surgical site infection is unknown. Many studies consider obesity as a marker or possible confounder for associated comorbidities with a greater association to risk of infection such as diabetes or malnutrition. BMI has been used as a practical tool for measuring and classifying obesity, but it is not able to discriminate confounders and does not consider the distribution of body fat. For these reasons, researchers begin to question BMI as an appropriate tool for evaluating outcomes. Furthermore, in our study population, it is not always easy to assess BMI. Many patients have not been properly measured and weighed for long periods of time. Information is usually assessed by family members and tends to be an inaccurate estimate. This could imply another drawback for using BMI.

To the best of our knowledge, there are no previous papers that associate local subcutaneous measurements with surgical site infection in patients with femoral fractures. In general surgery, Lee et al. found that abdominal subcutaneous fat is an independent predictor of superficial incisional SSI after midline laparotomy, and Fujii et al. also reported an increased risk of SSI in patients with greater subcutaneous fat thickness undergoing elective colorectal resection $[19,20]$. A similar study performed in elective lumbar spine surgery shows the utility of local subcutaneous fat thickness as a simple independent risk assessment tool for surgical site infection [16]. In cervical spine fusion procedures, Mehta et al. demonstrated that the thickness of subcutaneous fat at the surgical site is a factor in the development of surgical site infection [21]. Results of our study show an association between the measurement of subcutaneous tissue at the hip and the risk of SSI in elderly patients with a hip fracture.

SRM at the hip is a useful tool for detecting patients at an increased risk of surgical site infection. The problem with this measure is that it does not allow for long-term prevention strategies, such as weight loss, to decrease the soft tissue around the hip, especially in elderly hip fracture patients in which time to surgery is critical. However, SRM could be very useful in elective surgery patients helping the clinician evaluate the risk of infection and allowing for additional and differential prevention methods. We consider it could be interesting to further study local subcutaneous measurements in patients undergoing elective hip surgery and possible relationships between these measurements, surgical approach, and SSI.

Regarding fat distribution around the hip and surgical approaches in elective hip surgery, a recent study by Sprowls et al. described a greater soft tissue thickness in lateral hip incisions compared to anterior incisions [22]. This could potentially favor anterior approaches if an association was established with fewer SSIs. 
Multiple explanations can be considered as to why an increased local fat distribution at the hips is associated with an increased risk of SSI. It is possible that the simple fact of having an increased physical space may create technical difficulties for surgeon's leading to an increase in surgical time and subsequent infection. Toma et al. and Pevzner et al. have described pharmacokinetic variations in adipose tissue that could jeopardize minimal inhibitory concentrations for adequate antibiotic prophylaxis $[11,12]$.

Limitations of our study include the retrospecttive design and a relatively small sample size. We had to use immediate postoperative radiographs for the measurement of distances as we did not have access to preoperative radiographs for all patients. Radiographs were taken in a supine position which could have an influence in exact measurements. Some potential drawbacks to the SRM could include patient positioning, rotation of the pelvis, the orientation of the beam, magnification errors and morphology of the greater trochanter.

Our findings suggest an association between local SRM and surgical site infection. The fact that our study includes a relatively small sample size from a single geographic location may compromise external validity, for this reason, we believe there is a need for further study in this area of interest.

\section{Conclusions}

Results of our study suggest an association between local fat at the hip and the risk of SSI in elderly patients with surgically treated hip fractures. SRM may be a helpful tool for evaluating the risk of SSI in elderly hip fracture patients. However, further studies are necessary to confirm our results and to further evaluate its usefulness in patients after elective total hip replacement surgery.

\section{Abbreviations}

SSI: surgical site infection; SRM: subcutaneous radiographic measurement; THA: total hip arthroplasty; OR: odds ratio; CI: confidence interval; IM: Intramedullary; DHS: dynamic hip screw; HA: hemiarthroplasty; ASA: American Society of Anesthesiologists; MSSA: Methicillin-Susceptible Staphylococcus aureus; MRSA: Methicillin-resistant Staphylococcus aureus; ICC: Intraclass correlation coefficient; BMI: body mass index.

\section{Acknowledgements}

Martí Bernaus: Conceptualization, Data Curation, Formal Analysis, Investigation, Resources, Validation, Visualization, Writing- original draft. Francesc Anglès: Conceptualization, Investigation, Project administration, Supervision, Writing -review, and editing. Berta Escudero: Data Curation, Formal Analysis, Investigation, Validation, Visualization, Writing -review, and editing. Margarita Veloso: Conceptualization, Formal Analysis, Resources, Writing -review, and editing. Alfredo Matamala: Conceptualization, Investigation, Project administration, Resources, Supervision, Writing -review, and editing. Lluís Font-Vizcarra: Conceptualization, Data Curation, Formal Analysis, Investigation, Methodology, Project administration, Supervision, Visualization, Writing -review, and editing.

\section{Competing Interests}

The authors have declared that no competing interest exists.

\section{References}

1. Culver DH, Horan TC, Gaynes RP, Martone WJ, Jarvis WR, Emori TG, et al. Surgical wound infection rates by wound class, operative procedure, and patient risk index. National Nosocomial Infections Surveillance System. Am J Med. 1991 Sep 16;91(3B):152S-157S.

2. de Jong L, Klem TMAL, Kuijper TM, Roukema GR. Factors affecting the rate of surgical site infection in patients after hemiarthroplasty of the hip following a fracture of the neck of the femur. Bone Joint J. 2017 Aug 31; 99-B(8):1088-94.

3. Kempegowda H, Richard R, Borade A, Tawari A, Graham J, Suk M, et al. Obesity Is Associated With High Perioperative Complications Among Surgically Treated Intertrochanteric Fracture of the Femur. J Orthop Trauma. 2017;31(7):352-7.

4. Ovaska MT, Mäkinen TJ, Madanat R, Huotari K, Vahlberg T, Hirvensalo E, et al. Risk Factors for Deep Surgical Site Infection Following Operative Treatment of Ankle Fractures. J Bone Jt Surgery-American Vol. 2013 Feb 20;95(4):348-53.

5. Edwards C, Counsell A, Boulton C, Moran CG. Early infection after hip fracture surgery: RISK FACTORS, COSTS, AND OUTCOME. J Bone Jt Surg Br Vol. 2008 Jun 1;90-B(6):770-7.

6. Harrison T, Robinson P, Cook A, Parker MJ. Factors affecting the incidence of deep wound infection after hip fracture surgery. Bone Joint J. 2012 Feb 1;94-B(2):237-40.

7. Kaye KS, Schmader KE, Sawyer R. Surgical Site Infection in the Elderly Population. Clin Infect Dis. 2004 Dec 15;39(12):1835-41.

8. Dowsey MM, Choong PFM. Early outcomes and complications following joint arthroplasty in obese patients: a review of the published reports. ANZ J Surg. 2008 Jun;78(6):439-44.

9. Dowsey MM, Choong PFM. Obesity is a major risk factor for prosthetic infection after primary hip arthroplasty. Clin Orthop Relat Res. 2008 Jan 3;466(1):153-8.

10. Chee $\mathrm{YH}, \mathrm{Teoh} \mathrm{KH}$, Sabnis BM, Ballantyne JA, Brenkel IJ. Total hip replacement in morbidly obese patients with osteoarthritis: RESULTS OF A PROSPECTIVELY MATCHED STUDY. J Bone Jt Surg - Br Vol [Internet]. 2010 Aug 1;92-B(8):1066-71.

11. Toma O, Suntrup P, Stefanescu A, London A, Mutch M, Kharasch E. Pharmacokinetics and tissue penetration of cefoxitin in obesity: implications for risk of surgical site infection. Anesth Analg. 2011 Oct; 113(4):730-7.

12. Pevzner L, Swank M, Krepel C, Wing DA, Chan K, Edmiston CE. Effects of maternal obesity on tissue concentrations of prophylactic cefazolin during cesarean delivery. Obstet Gynecol. 2011 Apr; 117(4):877-82.

13. Kabon B, Nagele A, Reddy D, Eagon C, Fleshman JW, Sessler DI, et al. Obesity decreases perioperative tissue oxygenation. Anesthesiology. 2004 Feb; 100(2):274-80.

14. Hopf HW, Hunt TK, West JM, Blomquist P, Goodson WH, Jensen JA, et al. Wound tissue oxygen tension predicts the risk of wound infection in surgical patients. Arch Surg. 1997 Sep; 132(9):997-1004

15. Heymsfield SB, Cefalu WT. Does Body Mass Index Adequately Convey a Patient's Mortality Risk? JAMA. 2013 Jan 2; 309(1):87.

16. Lee JJ, Odeh KI, Holcombe SA, Patel RD, Wang SC, Goulet JA, et al. Fat Thickness as a Risk Factor for Infection in Lumbar Spine Surgery. Orthopedics. 2016 Nov $1 ; 39(6):$ e1124-8.

17. Tsukayama DT, Goldberg VM, Kyle R. Diagnosis and management of infection after total knee arthroplasty. J Bone Joint Surg Am. 2003; 85-A Suppl: S75-80.

18. de Vries LM, Neve WC, Steens J. Prosthesis retention after an infected hip prosthesis: hip fractures versus primary total hip prosthesis, data from 1998-2015. J Bone Jt Infect. 2018 ;3(3):118-22. 
19. Lee JS, Terimanian MN, Tishberg LM, Alawieh AZ, Harbaugh CM, Sheetz $\mathrm{KH}$, et al. Surgical Site Infection and Analytic Morphometric Assessment of Body Composition in Patients Undergoing Midline Laparotomy. J Am Coll Surg. 2011 Aug; 213(2):236-44.

20. Fujii T, Tsutsumi S, Matsumoto A, Fukasawa T, Tabe $Y$, Yajima R, et al. Thickness of Subcutaneous Fat as a Strong Risk Factor for Wound Infections in Elective Colorectal Surgery: Impact of Prediction Using Preoperative CT. Dig Surg. 2010; 27(4):331-5.

21. Mehta AI, Babu R, Sharma R, Karikari IO, Grunch BH, Owens TR, et al. Thickness of Subcutaneous Fat as a Risk Factor for Infection in Cervical Spine Fusion Surgery. J Bone Jt Surgery-American Vol. $2013 \mathrm{Feb} 20$; 95(4):323-8.

22. Sprowls GR, Pruszynski JE, Allen BC. Distribution of Subcutaneous Fat Around the Hip in Relation to Surgical Approach for Total Hip Arthroplasty. J Arthroplasty. 2016;31(6):1213-7. 